\title{
COMPETENCIAS DEL GERENTE EDUCATIVO EN INSTITUCIONES EDUCATIVAS DE RIOHACHA, COLOMBIA
}

\section{Competences of the educational manager in educational institutions of Riohacha, Colombia}

\section{Vianet Calle*}

\section{RESUMEN}

El presente estudio estuvo dirigido a analizar las competencias del gerente educativo en Instituciones Educativas de Riohacha, Colombia. Teóricamente, estuvo sustentada por Acosta y Finol (2015), Moreno (2012), Tobón (2006), García y Vaillant (2010), Aldape (2008), Zabalza (2007) entre otros En cuanto a la metodología, el tipo de investigación fue descriptiva, con un diseño no experimental y transversal y de campo, cuya población estuvo constituida por seis (6) directivos y noventa y tres (93) docentes de las instituciones educativas privadas de Riohacha, Colombia cuyo nombre son: Centro Educativo Enmanuel, Colegio Marcelo Graziosi y Gimnasio Campo David. En conclusión se evidencia que para los directores las competencias académicas, administrativas, comunicativas y humano-sociales, son puestas en práctica en las instituciones casi siempre, mientras que para los docentes dichas competencias casi nunca son demostradas por el personal directivo, estas divergencias indican una sentida necesidad de mejoramiento de las competencias del gerente educativo.

Palabras clave: Competencias del gerente educativo, académicas, administrativas, humano - sociales, comunicativas.

RECIBIDO: Octubre 2018

ACEPTADO: Junio 2019

\section{ABSTRACT}

The present study was aimed at analyzing the competences of the educational manager in Educational Institutions of Riohacha, Colombia. Theoretically, it was supported by Acosta and Finol (2015), Moreno (2012), Tobón (2006), García and Vaillant (2010), Aldape (2008), Zabalza (2007), among others. Regarding methodology, type The research was descriptive, with a non-experimental, transversal and field design, whose

\footnotetext{
* Docente de Aula de Institución Educativa y Catedrática Universidad de la Guajira-Colombia. Magister en Desarrollo Empresarial. Trabajadora Social. Especialista en Educación Cultura y Política. Actualmente desarrollando II semestre de doctorado Universidad Experimental del Libertador-Venezuela. Colombia. Correo electrónico: vianetcalle@ hotmail.com
} 


\section{Vianet Calle}

Telos Vol. 21, No. 3 (2019). 564-590

population consisted of six (6) managers and ninety-three (93) teachers from the private educational institutions of Riohacha, Colombia whose name is: Enmanuel Educational Center, Marcelo Graziosi School and David Campo Gym. In conclusion it is evident that for the directors the academic, administrative, communicative and human-social competences are practiced in the institutions almost always, while for the teachers such competences are almost never demonstrated by the directive personnel, these divergences indicate a felt need to improve the skills of the educational manager.

Keywords: Teacher qualifications, academic, administrative, human - social, communicative.

\section{Introducción}

El educador del siglo XXI, es visto como un profesional atento a todas las posibilidades para hacer atractivo el proceso educativo. En este orden, debe ser capaz de analizar, comparar, explicar, discernir críticamente su propia praxis pedagógica, en busca de orientar a todos los actores al logro de competencias necesarias para la inserción en la sociedad en permanente evolución en diferentes contextos. Actualmente, en el mundo la vertiginosidad de las transformaciones científicas y tecnológicas han influido en el desarrollo de la ciencia y el conocimiento, convirtiéndose en fuerzas directas en las sociedades industrializadas, materializadas y automatizadas, estableciéndose como elementos relevantes en los procesos educativos.

Bajo esta perspectiva, la sociedad del conocimiento ha generado una serie de cambios que han llevado al ser humano al establecimiento de nuevas formas de interrelacionarse con sus semejantes y con el entorno, donde prima el poder, el cual no está sólo en el ámbito económico, sino que va más allá, hacia el dominio del conocimiento, el cual constituye una ventaja competitiva, para el desarrollo o desenvolvimiento a nivel educativo.

Aunado a la idea anterior, Acosta y Finol (2015), mencionan que en la actualidad cada vez es más alto el nivel educativo que necesitan los profesionales para participar en la sociedad y resolver problemas de carácter práctico. Es por ello, que es imprescindible que la educación potencie el desarrollo de competencias, las cuales las define como un conjunto de conocimientos, habilidades, destrezas, actitudes, aptitudes y valores que forman parte 
Competencias del gerente educativo en instituciones educativas de Riohacha, Colombia.

activa en el desempeño responsable y eficaz de un individuo para el ejercicio de un conjunto de actividades cotidianas dentro de un contexto determinado, dichas competencias deben ser direccionadas a facilitar el ingreso y permanencia eficaz de un individuo en el campo laboral para desarrollarse efectivamente en la sociedad.

En este contexto, Aldape (2008), destaca las competencias académicas, administrativas y humanas, las cuales juegan un papel relevante en los procesos educativos. En consecuencia, se exige el desarrollo de dichas competencias para que puedan adaptarse a ese acelerado avance en la ciencia y la tecnología en la sociedad actual, es por ello, que la labor educativa debe enfocarse hacia un paradigma que permita afrontar las nuevas exigencias a nivel económico, político y social, lo que implica la formación de personas competentes que puedan adaptarse a la sociedad del siglo XXI.

Durante los últimos años, se ha originado a niveles mundiales algunos criterios para la evaluación del desempeño docente, derivados del enfoque por competencias en educación; algunas experiencias han contribuido notablemente a la expansión del enfoque basadas en actitudes. Por su parte, el Parlamento Europeo (2010), ha establecido un marco de referencia con once competencias clave que debe desarrollar el docente del siglo XXI, las cuales son: comunicación en la lengua materna y lenguas extranjeras, competencia matemáticas y competencias básicas en ciencia y tecnología; competencia digital, aprender - aprender, competencias interpersonales, interculturales, humano - sociales, administrativas, competencia cívica, espíritu emprendedor y expresión cultural, entre otras.

Por su parte, Semenov (2005) teniendo en cuenta la inclusión de la ciencia y la tecnología en la vida de las personas considera importante la posesión de unas competencias desde el ámbito educativo donde los docentes y directores, como pilares fundamentales en el desarrollo de las nuevas generaciones, deben poseer ciertas competencias como son las académicas, administrativas, humano - sociales.

A nivel latinoamericano, González y Ramírez (2011), refiere que la búsqueda de puntos comunes de referencia entre los distintos programas se centra en las 


\section{Vianet Calle}

Telos Vol. 21, No. 3 (2019). 564-590

competencias expresadas en resultados de aprendizaje para respetar la diversidad, la libertad, el manejo de conflictos y la autonomía de cada institución, así como para identificar los distintos papeles de los actores más importantes: los profesores, los estudiantes, además, las competencias, objeto de los programas educativos, representan una combinación dinámica de conocimientos, comprensión, habilidades y capacidades.

En el mismo orden de ideas, se infiere que las competencias son un saber hacer que lleva a un desempeño eficiente, el cual puede demostrarse mediante ejecuciones observables. Es decir, se trata de una capacidad para resolver problemas que se aplica de manera flexible y pertinente, adaptándose a cualquier contexto y a las demandas que plantean situaciones diversas presentadas en el entorno.

En criterio de, Belcher \& Braine (1995, p. 384), acotan que estos cambios "repercuten sobre el desempeño y desarrollo, al modificar el comportamiento del individuo para elevar el aprovechamiento de los recursos, mejorar la calidad y productividad de los servicios educativos mediante la adecuada combinación de acciones gerenciales, ante conflictos organizacionales". De ese modo, el gerente educativo, se forma, actualiza y desenvuelve como elemento sustantivo que impulsa la productividad y la calidad de la institución.

Así, las competencias académicas, administrativas y humano - sociales, son aspectos vitales en el desarrollo y desenvolvimiento del gerente educativo, puesto que a través de ellas, no sólo prepara a las nuevas generaciones para ser competitivos en un mercado que así lo exige, sino también le permite a él, desde su rol de educador, ser un profesional abierto a un mundo acelerado de cambios, donde sólo sobrevive quien posea las competencias requeridas en una sociedad enmarcada en el conocimiento y la tecnología.

Desde esa perspectiva, para el desarrollo efectivo de competencias adecuadas en los centros educativos, los directivos y docentes proyectan cambios en su cultura de trabajo, con el fin de mejorar las diferencias de intereses u opiniones entre una o más partes sobre determinada situación, lo cual es importante para evitar que ocurran 
Competencias del gerente educativo en instituciones educativas de Riohacha, Colombia.

conflictos organizacionales que afecten la marcha eficiente de las actividades en el entorno escolar.

Según Moreno et al., (2011), consideran que hoy en día el cambio educativo que se está promoviendo en Colombia, requiere de la transformación de la praxis del profesional de la docencia y de su formación, ya que hasta los momentos los que se han venido ejecutando no han respondido a las actuales demandas que surgen de las innovaciones pedagógicas y educativas de la actualidad. Cabe destacar que el sistema educativo colombiano, se ha planteado como reto introducir ciertos cambios en su organización, siendo uno de ellos los estilos gerenciales para que respondan a la demanda actual de la gerencia educativa.

En opinión de Pozner (2000), tanto directivos como docentes tienen capacidad para organizar, administrar y convocar a reuniones donde participen los distintos actores sociales para la realización de planes, programas, actividades o tareas. Es decir, los mismos se encuentran aislados de su comunidad, de la sociedad y de la institución educativa, en cuanto prevalece una actitud pasiva, sólo desarrollan el papel de gestores escolares, deslindados de la realidad cotidiana de la organización. De ello, surge la falta de interés, participación, la integración, dificultando así, las acciones pedagógicas.

Considerando el criterio de Ávila (2013), plantea que todo gerente debe dar soluciones a problemas y esto un proceso estructurado con una finalidad única, dentro de un marco cooperativo que permite atender las necesidades existentes, compartiendo las alternativas más convenientes para reorientar o fortalecer los objetivos planteados dentro de un proceso participativo. De acuerdo a lo anterior, pueden tomarse decisiones situacionales, de tal forma que permitan reorientar los escenarios previstos, los imprevistos, considerando los procesos que la sistematizan u ordenan, además de la existencia de personas ganadas, y otros que ejercen resistencia hacia los objetivos organizacionales que se planifican en las instituciones educativas para desarrollar el potencial.

Ante lo expuesto con anterioridad, González y Ramírez (2011) mencionan que los consejos de docentes se centran en suministrar información, solución a manejo de 


\section{Vianet Calle}

Telos Vol. 21, No. 3 (2019). 564-590

conflictos, pero no estimulan la búsqueda de solución a los problemas que presentan los docente, los estudiantes y todo personal adscrito a las instituciones educativas, la dirección no realiza una verdadera evaluación de los resultados cualitativos y cuantitativos obtenidos al concluir cada lapso o año escolar, ni se aplican control y seguimiento de las acciones planificadas e implementadas.

Desde este punto de vista, resulta evidente que el director en su función de gestor de las decisiones académicas de las instituciones de Riohacha, Colombia, debe propiciar condiciones en los planteles para que los docentes coordinen el tránsito de los estudiantes por la escolaridad, bajo ciertas exigencias curriculares e instruccionales, que apoyen a la vez, el desempeño del docente.

En este contexto, según visitas guiadas a las instituciones educativas de Riohacha, se pudo observar que existe un déficit en el desarrollo de ciertas competencias como son las académicas, administrativas, humano - sociales, por parte de los directivos y docentes, lo cual podría traer como consecuencia la falta de unificación de criterios para la toma de decisiones, el manejo inadecuado de la tecnología, elaboración de cuadros estadísticos, actividades de evaluación y control, Falta de organización del material e informaciones, interrupciones constantes en las reuniones del consejo técnico, situaciones problemáticas al momento de interrelacionarse, entre otros.

En este marco de ideas, de no llegar a solucionarse este tipo de situaciones se presentarían problemas más agraviantes o perjudiciales, que originarían conflictos severos en el sistema, dentro de la estructura académica, administrativa, comunicativas y humano - sociales que afectan el desempeño del docente y de los directores evitando que sus acciones sean competentes.

Por la situación antes planteada, se hace necesario analizar las competencias del gerente educativo en instituciones educativas de Riohacha, Colombia. A tal efecto, hay que formar un nuevo docente con unas competencias relacionadas al proyecto de vida, con una consciencia crítica, participativa, creativa, humanista que conozca la realidad 
Competencias del gerente educativo en instituciones educativas de Riohacha, Colombia.

existente de su entorno escolar y que tenga sentido de pertenencia como mediador de conocimiento en diversas áreas, siendo un facilitador que desempeñe las competencias de manera correcta, creando un clima ideal en su sitio de trabajo. Este artículo está estructurado con los siguientes puntos: competencias del gerente educativo, tipos de competencias: académicas, administrativas, comunicativas y humano - sociales, metodología, resultados, reflexiones finales y referencias bibliográficas.

\section{Competencias del Gerente Educativo}

Desde el punto de vista de Tobón (2006), las competencias “son los procesos generales contextualizados, referidos al desempeño de la persona dentro de una determinada área del desarrollo humano". A su vez, las competencias del gerente educativo son una orientación de cómo se desempeña el individuo como tal, en diversas áreas como la académica, administrativa, comunicativa y social en busca de la idoneidad en la realización de actividades y resolución de problemas. Por tal motivo, estas se apoyan en los indicadores de logro como una manera de ir estableciendo su formación en etapas.

El mismo autor indica que las actuaciones integrales que poseen los docentes y gerentes, el proyecto ético de vida y el desarrollo de competencias de los estudiantes, están relacionadas por medio de la movilización articulada del saber ser, saber hacer, saber conocer y el saber convivir. Por otra parte, Zabalza (2007), plantea que las competencias del gerente "son el conjunto de conocimientos que necesitan para desarrollar una actividad". Cada actividad suele exigir la presencia de un número variado de competencias que pueden posteriormente ser desglosadas en unidades más específicas, es decir, se puede decir brevemente que las competencias del gerente educativo es la actitud de cómo compete y ejerce sus conocimientos el gerente en cualquier situación.

Así mismo, para García y Vaillant (2010), las competencias son el conjunto de saberes técnicos metodológicos sociales y participativos que se actualizan en una situación y en un momento particular, es decir, es un saber hacer completo. Por su parte, 


\section{Vianet Calle}

Telos Vol. 21, No. 3 (2019). 564-590

los mismos autores mencionan que la competencia del gerente educativo son capacidades que tienen los directores para dar solución a problemas, situaciones, entre otros, produciendo un nuevo conocimiento, además, también es considerada una formación compleja y holística, que se conforma a partir de la integración funcional de cualidades, atributos o elementos establecidos en el sujeto.

Así, una actuación competente incluye conocimientos, habilidades, actitudes y valores, pero el todo no es igual que la suma de sus partes; el resultado es algo más completo y diverso que estos tres ingredientes reunidos. Por tal razón, la persona competente debe responder empleando toda su ser y hacer una lectura correcta del contexto determinado; y es sólo en ese marco que se pueden desarrollar y potenciar (Moreno 2012). En este orden, el término "competencia" conlleva saber y saber hacer, teoría y práctica, conocimiento y acción, reflexión y acción; esto va a representar una transformación en el conocimiento: del saber qué al saber cómo.

Dentro de esta investigación se toma en consideración la relación que existe entre lo que explica Tobón (2006) sobre las diversas actuaciones para el desarrollo de distintas competencias de los docentes y directores, además de lo que explica Zabalza (2007) indicando que las competencias del gerente educativo se irán desarrollando a medida de que evolucionan sus habilidades y conocimientos a diferencia de García y Vaillant (2010) que expone que las competencias se actualizan en un momento determinado y especifico.

En este orden, el gerente educativo maneja extensos conocimientos donde desarrolla las habilidades pertinentes para el cumplimiento de sus responsabilidades y en las competencias para solucionar cualquier problema que se pueda presentar en todas las acciones ejecutadas en la organización educativa. En tal sentido, se infiere que las competencias del gerente son habilidades o aptitudes que desarrolla a lo largo de su 
Competencias del gerente educativo en instituciones educativas de Riohacha, Colombia.

trayectoria profesional, presenta capacidad para hacer, promoviendo conocimiento en contextos establecidos, en el trabajo, para integrar saberes o solucionar problemas.

Para finalizar, en las instituciones educativas de Riohacha, Colombia, considerando la teoría de Moreno (2012) cualquier gerente educativo debe tener capacidad para planificar, orientar, organizar, dirigir, entre otras, actividades que fomenten la integración de los grupos de trabajo para beneficio de todos los actores del proceso de enseñanza y aprendizaje, mediante un diálogo ameno, participativo, unificando criterios para mejoramiento de la acción gerencial y comunicacional.

\section{Tipos de Competencias}

Según Moreno (2012), menciona que los tipos de competencias pueden ser ordenados siguiendo diversas clasificaciones, casi tantas como autores. En algunas ocasiones también diversas clases o divisiones. A su vez, es muy usual la ordenación en cuatro ámbitos: académicas, administrativas, comunicativas y humano - sociales, incluyendo dos más la participativas y transversales en el contexto institucional.

Por otra parte, González y Ramírez (2011), indica que los tipos de competencias son esenciales para formar la metodología propia de cada empresa organización y depende del manejo de información, el área, los conocimientos que posean los gerentes, docentes o cualquier persona que mejoren su desempeño mediante la comunicación eficaz en pro de desarrollar las habilidades y que fomenten el dialogo en las organizaciones.

En criterio de Martínez (2005), los tipos de competencias que se encuentran en la actualidad son de amplia variedad, de conformidad con la institución que las requiere, las formas o las promueve. Ante este panorama se deben analizar las variantes que existen tanto para la gerencia profesional. Estas a su vez, se distinguen por ser herramientas para alcanzar un fin determinado, en esta perspectiva, son una 


\section{Vianet Calle}

Telos Vol. 21, No. 3 (2019). 564-590

combinación de habilidades metodológicas que favorecen a las competencias profesionales.

Para finalizar los tipos de competencias del gerente educativo están relacionados con las diversas variedades de categorías existentes en los individuos que ocupan cualquier cargo, estas ayudan a establecer el perfil de cada persona mostrando sus capacidades, habilidades, aptitudes para el desarrollo de proyectos organizacionales en pro de mejorar el desempeño. En este orden, en la actualidad tanto los directivos como docentes consideran relevante las competencias en las personas para negociar, interactuar con responsabilidad y honestidad, trabajando en equipo, mediante una comunicación eficaz establecida en el respeto mutuo.

\section{Competencias Académicas}

Según Aldape (2008), las competencias académicas son aquellas que le permitirán al docente dominar los conocimientos y habilidades específicas sobre su materia de especialidad, es decir, los métodos, los equipos y las tecnologías para la enseñanza aprendizaje. Además, también facilitan el trabajo en el ámbito educativo. En este entorno, el docente adquiere una visión sistémica para entender la institución como un todo y percibir un impacto de sus decisiones en los diversos subsistemas en el sistema social, debido a las conexiones entre distintos elementos, permitiendo la integración de los grupos de trabajo, una visión sistémica para mejorar la toma de decisiones.

Para Moreno (2012), la competencia académica define el conjunto de conocimientos, habilidades, capacidades y conductas que posee una persona, que le permiten la realización exitosa de una actividad. Es decir, hace que los individuos sean competentes para realizar una actividad, plan, proyecto, entre otros, teniendo éxito en 
Competencias del gerente educativo en instituciones educativas de Riohacha, Colombia.

la misma. Desde este contexto, las competencias no tienen por qué pertenecer a una rama de estudio específica.

En este orden, las competencias académicas se pueden conceptualizar como todo lo que un gerente o docente debe saber, relacionado esto con una disciplina particular y su aplicación significativa para crear conocimiento a partir de lo adquirido. A su vez, Acevedo (2007), menciona que en todas las áreas los referentes para seleccionar este conocimiento son de tres tipos: El referente universal de la disciplina, el de la cultura regional y el referente de los intereses y motivaciones individuales.

En tal sentido, estas deben ser aplicadas en la parte administrativa o a la gerencia de las organizaciones para mejorar las relaciones entre el personal. Así, se infiere que las competencias académicas dependen de los intereses de los grupos, de la cultura en un contexto determinado. Las mismas están relacionadas con las competencias laborales en una organización. En tal sentido, una formación académica basada en competencias, está relacionada con el desempeño de gerentes y docentes que exige el mundo actual de acuerdo a las necesidades de las personas o equipos de trabajo.

Para finalizar, actualmente, el ámbito escolar demanda personas competentes para el desempeño de funciones, con un perfil que permita tener sentido de pertenencia y vocación, ante esto, para ser competente en el trabajo deberá adquirir conocimientos, desarrollando diversas habilidades y actitudes en el entorno humano, desde un liderazgo efectivo, para la resolución de situaciones problemáticas.

\section{Competencias Administrativas}

En criterio de Aldape (2008), las competencias administrativas constituyen los conocimientos y habilidades específicas que contribuyen a enlazar las actividades docentes con las demandas de la administración de la institución del entorno de la misma. Además, las nuevas tendencias vislumbran exigencias del mundo global de este siglo que van más allá de las funciones de planificación, organización, dirección y 


\section{Vianet Calle}

Telos Vol. 21, No. 3 (2019). 564-590

control de planes o proyectos, dependiendo de las actividades que desarrolle el gerente y del rol que desempeñe dentro de la organización.

Según la autora antes mencionada, el no poseer dichas competencias representa una desventaja competitiva, porque se tiende a desmejorar la calidad de las acciones, lo que contribuye al bajo rendimiento de éste y por ende, a una disminución en el compromiso para el logro de las metas propuestas de la institución, por no tener unificación de criterios entre las partes.

Cabe destacar, que esta competencia le permite al gerente desde su práctica educativa una adecuada organización de su actividad pedagógica y administrativa, ya que le proporciona en primer lugar, planificar su trabajo, lo que conlleva al aporte de las metas a seguir y por ende, a una organización de las actividades sin alejarse de los objetivos propuestos, visualizando las fortalezas y debilidades, y en segundo lugar, está la revisión de las actividades planeadas, ejerciendo un control sobre ellas, para lograr finalmente los resultados esperados.

Según Alles (2015), las muestra de un modo genérico que a su vez, suele entenderse la competencia laboral como las actitudes, los conocimientos y las destrezas que permiten desarrollar exitosamente un conjunto integrado de funciones administrativas de acuerdo a criterios de desempeño considerados idóneos en el medio laboral y que por ende se encuentra relacionada con la comunicación organizacional.

Además, se identifican en situaciones reales de trabajo y se las describe agrupando las acciones productivas en áreas de competencia (funciones más o menos permanentes), especificando para cada una de las tareas los criterios de realización a través de los cuales se puede evaluar su ejecución en un tiempo determinado, dependiendo del contexto o ambiente donde se desarrolla.

Para, Prego (2007), como aquellas cualidades personales de los individuos, permitiendo observar el desempeño excelente en un entorno cambiante relacionado con muchas funciones. En este orden, la capacidad de aprendizaje, la flexibilidad, habilidad 
Competencias del gerente educativo en instituciones educativas de Riohacha, Colombia.

y capacidad de adaptación son más importantes en este sentido que el conocimiento o la praxis en el manejo de un determinado lenguaje de programación o una herramienta informática específica.

Por otra parte, el Parlamento Europeo (2010), se refiere a la competencia administrativa como la organización en el trabajo, atendiendo en su parte normativa a la planificación, coordinación y gestión de la calidad en la institución; para lo cual el docente debe tener unos conocimientos, habilidades y destrezas propias. También son conocidas como competencias de gestión.

De acuerdo a, Robbins (2009), la competencia administrativa se refiere a las capacidades inmersas en los comportamientos de quien dirige, todo ello, acompañado por una serie de habilidades críticas para administrar, dentro de las cuales están 1) las habilidades generales y 2) las específicas: La primera involucra otras como las conceptuales, las interpersonales, las técnicas y las políticas; y la segunda que incluye el manejo de una serie de aspectos como: el control del entorno y sus recursos, la organización y coordinación, el manejo de información, entre otros.

Acorde a lo anterior, la competencia administrativa muestra cierta complejidad, puesto que incluye una serie de sub - competencias como son las generales, contenida en las siguientes habilidades: a) la conceptual que se refiere a la capacidad para analizar situaciones complicadas, b) las interpersonales que involucra la capacidad de enseñar y para trabajar en equipo y también la motivación que se pueda ejercer sobre los demás,

c) Técnica que está relacionada con la comprensión de procesos y productos en la organización.

Se puede acotar que la competencia administrativa es una habilidad de suma importancia para el docente y directores, puesto que le permite direccionar sus acciones pedagógicas en función de unas metas previamente trazadas, para lo cual debe encaminar todo su esfuerzo. Además, el gerente no sólo requiere de esta competencia a nivel personal, sino también, en quehacer pedagógico para así poder preparar de manera conveniente sus actividades académicas y darle un rumbo efectivo al desarrollo de su 


\section{Vianet Calle}

Telos Vol. 21, No. 3 (2019). 564-590

trabajo escolar, así preverá posibles situaciones que de alguna manera puedan intervenir en la ejecución de sus actividades. Además, al poseer dicha competencia este ejecutará las acciones pertinentes para el buen desempeño de su labor.

Considerando lo expuesto, las competencias administrativas están referidas a los procesos o funciones que ejecuta el docente en su jornada escolar, como son la planificación, organización, diagnóstico, evaluación, entre otras, las cuales permiten llevar un control de todo el trabajo realizado en el ámbito educativo, facilitando la ejecución de actividades. Finalmente, estas son relevantes en las instituciones educativas de Riohacha, Colombia.

\section{Competencias Comunicativas}

Según Tobón (2011), la competencia comunicativa es el "conjunto de procesos y conocimientos de diversos tipos lingüísticos, sociolingüísticos, estratégicos y discusivo, que el hablante - oyente - escritor - lector, deberá poner en juego para producir, comprender discursos adecuados a la situación y al contexto de comunicación y al grado de formalización". Se interpreta que a través de las cualidades, valores, estimulaciones y sus incomparables escenarios de interacción, desde este contexto, Niño (2009), las refiere como un saber especifico y el saber aplicarlo, saberes basados en conjuntos de conocimientos, aptitudes y valores. De los miembros de un grupo social, que las habilita para realizar actos comunicativos eficientes.

Además, la competencia comunicativa se puede denominar como el término más frecuente para la capacidad comunicativa de un ser humano, conocida como la capacidad que comprende tanto el conocimiento de la lengua como la experiencia para utilizarla. Según el aporte de Arconada, (2006), indica que la competencia comunicativa no consiste solo en tener la competencia lingüística que permita hablar una lengua con 
Competencias del gerente educativo en instituciones educativas de Riohacha, Colombia.

arreglo a sus leyes gramaticales, sino también en usar habilidades y conocimientos en diferentes escenarios de comunicación.

Según Moreno et al (2011), la competencia comunicativa en el docente y el gerente está demarcada por la capacidad para gestionar de manera didáctica la información y las destrezas que desean construir con los estudiantes y con todo el personal que labora en la organización. Lo que implica que éste tenga la habilidad de convertir las ideas o contenidos disciplinares en mensajes comprensibles.

Aunado a lo anterior, esta competencia comprende, según el autor antes mencionado, los siguientes aspectos: a) Producción comunicativa, b) Refuerzo de la comprensibilidad, referida ésta, al manejo del mensaje a través de diferentes códigos y al otorgamiento de simplicidad, orden y brevedad del mismo. c) Organización interna de los mensajes, d) Connotación afectiva de los mensajes, la cual implica una estrategia para personalizarlos y así facilitar el nivel de atención de los alumnos.

Por otra parte, Gorrochotegui (2010), define a la competencia comunicativa como un elemento fundamental en la vida social del ser humano, la cual contiene elementos muy importantes como son: uso del lenguaje, postura, actitud corporal, gesto, tono, volumen de la voz, el ritmo del habla; que contribuyen a que se propicie el acto comunicativo de manera eficaz. Asimismo, considera que la competencia comunicativa, se puede ver afectada en el contexto educativo, cuando ésta se maneja dentro de una educación de estilo tradicional donde la repetición influye de manera negativa en el desarrollo de ésta.

Por tal razón, los gerentes deben desarrollar mediante el uso de estrategias adecuadas, la competencia comunicativa, donde el lenguaje es el instrumento del intercambio de ideas, éstas, dan paso al conocimiento, si hay atención, dedicación, interés y comprensión de los mensajes, facilitando la interacción entre los sujetos a 


\section{Vianet Calle}

Telos Vol. 21, No. 3 (2019). 564-590

través de la comunicación eficaz en todos los departamentos de la organización educativa.

En el concepto de Miranda, et al., (2009), señala que la competencia comunicativa posee dos elementos que se integran entre sí, que son el conocimiento de las reglas gramaticales y las reglas socioculturales de uso; y el otro elemento, referido a la habilidad del uso en la que intervienen aspectos como: la motivación, la confianza, la cortesía y la concentración, entre otros. No se puede olvidar que en todo proceso comunicacional, los aspectos socioculturales intervienen en el uso de la lengua, es decir, la manera como se expresan las personas, considerando los contextos y el momento donde se presentan. Claro está, los seres humanos deben ser los primeros interesados en comprender la información emitida, para ello necesitan decodificar los mensajes recibidos, logrando así una retroalimentación.

Según Palacino (2007), la competencia comunicativa se logra cuando se usa de manera adecuada un conjunto de conocimientos, habilidades y normas, las cuales son imprescindibles para comunicarse de una manera correcta teniendo en cuenta no sólo el contexto, sino también, la situación en que se da el acto comunicativo, donde intervienen tanto el emisor como los receptores del proceso.

Fajardo (2011), plantea el concepto de competencia comunicativa como el empleo efectivo del lenguaje en situaciones específicas de comunicación teniendo en cuenta las demandas del entorno. Asimismo, esta competencia necesita poseer otros conocimientos no lingüísticos, los cuales han de estar ligados con la comunicación verbal y en concordancia con aquellos aspectos sociales y de creencias; en otras palabras, las asociadas al factor cultural.

Según este autor, la competencia comunicativa se centra en la efectividad del lenguaje, dada por el manejo que el individuo haga de ésta, en el proceso de interacción con las demás personas en un espacio concreto, teniendo en cuenta que ésta va más allá del aspecto lingüístico y gramatical, pues el acto comunicativo debe darse de manera 
Competencias del gerente educativo en instituciones educativas de Riohacha, Colombia.

integral en todas las direcciones. En este mismo sentido, Hellín (2007), considera que la competencia comunicativa está compuesta por la suma de dos competencias muy importantes como son: la lingüística, en la que se observa el manejo de unos códigos que deberán ser decodificados, y por otro lado, la pragmática que aplica el uso práctico del lenguaje en contextos determinados entre los interlocutores que la practican.

En correspondencia a todo la idea mencionada con anterioridad, se puede apreciar que las competencias comunicativas se constituyen en una de las habilidades de mayor relevancia que necesita el docente, no sólo porque contribuye al desarrollo de su vida social, sino también, para el buen desempeño en su quehacer pedagógico, donde tiene que adaptarse a las exigencias de un mundo, sumergido en la tecnología, cuyo sistemas de información y comunicación demandan el manejo e inclusión de nuevos códigos lingüísticos para lo cual el docente debe estar preparado.

En este orden, es imprescindible reflexionar sobre el tipo de práctica oral que se esté estableciendo en el contexto educativo. Por tal razón, la comunicación es un medio para lograr la retroalimentación de las partes, haciendo transferencia de las situaciones reales o actos comunicativos existentes entre los actores del proceso educativo.

De esto, se puede afirmar que las competencias comunicativas están relacionadas con el proceso de comunicación que establecen los seres humanos al momento de interactuar ante cualquier situación que se presente, donde debe existir una retroalimentación para poder comprender lo que se está diciendo, mediante el uso del mismo código lingüístico.

\section{Competencias Humano - Sociales}

Según Aldape (2008), las competencias humano-sociales, son las que constituyen los conocimientos y habilidades específicas relacionadas con la capacidad del docente para trabajar armoniosamente con su grupo de clase, sus compañeros, padres de familia y cualquier otra persona involucrada en el logro de su meta Las competencias humanas son formas de caracterizar operativamente y con propósitos formativos, es una forma de conciencia y habilidad general, que es el resultado de la integración de conceptos, 


\section{Vianet Calle}

Telos Vol. 21, No. 3 (2019). 564-590

destrezas y actitudes y que le permite a la persona entender y transformar sus relaciones con la realidad, incluida ella misma.

Para Alles (2015), la competencia humana - social es el producto del dominio de conceptos, destrezas y actitudes; lo que quiere decir: ser competente significa que la persona tiene el conocimiento declarativo (la información y conceptos), en tal sentido, sabe lo que hace, por qué lo hace y conoce el objeto sobre el que actúa, desarrollándose en diversos contextos de las organizaciones. En este contexto, Villarini (2008), acota que ser competente está relacionado con la capacidad de ejecución, es decir el conocimiento procesal o las destrezas intelectuales para llevar a cabo la ejecución sobre el objeto. Finalmente, cuando un gerente educativo es competente tiene la actitud o disposición (conocimiento actitudinal) para querer hacer uso del conocimiento, actuando de forma correcta.

Las competencias humanas - sociales tiene características las cuales son aprendizajes comprensivos, resultado de la totalidad de experiencias educativas formales e informales; son características generales que la persona manifiesta en situaciones o escenarios como parte de su comportamiento; forman parte de una comunidad, además, se presentan en las cualidades valiosas de los individuos. Además, son capacidades generales que se desarrollan como parte del proceso de madurez, a partir del potencial humano para el aprendizaje, y ante los retos de la vida le muestran a la persona; una capacidad para llevar a cabo diversas tareas en una forma que es considerada como eficiente o apropiada.

Desde este punto de vista, en estos nuevos tiempos, se considera que para ser un buen gerente se requiere tener unas cualidades mínimas como persona, sobre todo cuando se refiere a un oficio tan importante como lo es ser docente (director encargado de una institución), aquí se pueden nombrar a la ética, la autoestima, la tolerancia, la 
Competencias del gerente educativo en instituciones educativas de Riohacha, Colombia.

motivación, la comunicación entre otras, las cuales son necesarias para que un gerente se relacione con sus docentes en cualquier contexto.

\section{Metodología}

Este artículo presentó un tipo de investigación descriptiva, mediante un diseño no experimental transversal y de campo. Asimismo, el estudio resulta descriptivo, debido al propósito del mismo, que no es más que exponer el evento estudiado, haciendo una enumeración detallada de sus características, de modo tal que en los resultados se pueden obtener dos niveles de análisis, dependiendo del fenómeno y el propósito del investigador. De lo anterior, resulta que el diseño bajo el cual se llevó a cabo el estudio fue no experimental transeccional, ya que el investigador observa los fenómenos tal y como ocurren naturalmente, sin intervenir en su desarrollo. Asimismo, el diseño es transversal o transeccional, pues los criterios u opiniones de los grupos estudiados son tomados en un momento dado (Hurtado, 2008).

La población quedó determinada por 6 directivos y 93 docentes de las instituciones educativas privadas de Riohacha, Colombia cuyo nombre son: Centro Educativo Enmanuel, Colegio Marcelo Graziosi y Gimnasio Campo David, considerando que presentan características similares como el grado de instrucción de los gerentes y docentes, cercanía de las mismas, nivel educativo al cual pertenecen. En este orden, se utilizó la observación por encuesta, mediante la elaboración de un cuestionario conformado con cinco (5) alternativas de respuesta, las cuales se mencionan a continuación: Siempre (S), casi siempre (CS), algunas veces (AV), casi nunca (CN) y nunca $(\mathrm{N})$. 


\section{Resultados}

Tabla 1. Competencias Académicas

\begin{tabular}{|l|l|l|l|l|l|l|l|l|l|l|}
\hline \multirow{3}{*}{ Alternativas } & \multicolumn{9}{|c|}{ Directores } & \multicolumn{7}{c|}{ Docentes } \\
& \multicolumn{1}{|c|}{$\begin{array}{l}\text { Item } \\
\mathbf{1}\end{array}$} & $\begin{array}{l}\text { Item } \\
\mathbf{2}\end{array}$ & $\begin{array}{l}\text { Item } \\
\mathbf{3}\end{array}$ & $\mathbf{X}$ & $\mathbf{\%}$ & $\begin{array}{l}\text { Item } \\
\mathbf{1}\end{array}$ & $\begin{array}{l}\text { Item } \\
\mathbf{2}\end{array}$ & $\begin{array}{l}\text { Item } \\
\mathbf{3}\end{array}$ & $\mathbf{X}$ & $\%$ \\
\hline Siempre & 0 & 0 & 0 & 0 & 0 & 0 & 0 & 0 & 0,0 & 0,0 \\
\hline Casi siempre & 5 & 4 & 3 & 4 & 67 & 8 & 7 & 0 & 5,0 & 5,4 \\
\hline Algunas Veces & 1 & 0 & 2 & 1 & 16,5 & 34 & 35 & 0 & 23,0 & 24,7 \\
\hline Casi nunca & 0 & 2 & 1 & 1 & 16,5 & 42 & 41 & 93 & 58,7 & 63,1 \\
\hline Nunca & 0 & 0 & 0 & 0 & 0 & 9 & 10 & 0 & 6,3 & 6,8 \\
\hline TOTAL & 6 & 6 & 6 & 6 & 100 & 93 & 93 & 93 & 93 & 100 \\
\hline
\end{tabular}

Fuente: Elaboración propia

Se observa en la Tabla 1, referida a las competencias académicas como una de las competencias que deben estar presentes en los gerentes, que la mayoría de los directores (67\%) considera que casi siempre realiza reuniones para captar la atención de los equipos de trabajo, elaborando proyectos para el éxito de la institución y proponiendo métodos de supervisión escolar, además, el 16,5\% restante algunas veces actúa de forma proactiva, mientras que otro $16,5 \%$ refirió que casi nunca.

Sin embargo, el 63,1\% de los docentes encuestados consideran que los directivos casi nunca se comportan como indican en el párrafo anterior, el 24,7\% opina que algunas veces lo hacen, seguidos de un $6,8 \%$ para quienes nunca demuestran competencias académicas y finalmente, el 5,4\% señaló que casi siempre muestran estas competencias.

Estos resultados revelan que según perciben los docentes, los directivos no se comportan acorde a su cargo gerencial, ya que discrepan de lo señalado por Moreno (2012), para quien la competencia académica define el conjunto de conocimientos, habilidades, capacidades y conductas que posee una persona, que le permiten la realización exitosa de una actividad. 
Competencias del gerente educativo en instituciones educativas de Riohacha, Colombia.

Tabla 2. Competencias Administrativas

\begin{tabular}{|c|c|c|c|c|c|c|c|c|c|c|}
\hline \multirow[b]{2}{*}{ Alternativas } & \multicolumn{5}{|c|}{ Directores } & \multicolumn{5}{|c|}{ Docentes } \\
\hline & $\begin{array}{c}\text { Item } \\
4 \\
\end{array}$ & $\begin{array}{c}\text { Item } \\
5 \\
\end{array}$ & $\begin{array}{c}\text { Item } \\
6 \\
\end{array}$ & $\mathrm{X}$ & $\%$ & Item 4 & $\begin{array}{c}\text { Item } \\
5 \\
\end{array}$ & $\begin{array}{c}\text { Item } \\
6 \\
\end{array}$ & $\mathrm{X}$ & $\%$ \\
\hline Siempre & 0 & 0 & 0 & 0 & 0 & 0 & 0 & 0 & 0,0 & 0,0 \\
\hline Casi siempre & 3 & 4 & 6 & 4,3 & 72 & 9 & 7 & 11 & 9,0 & 9,7 \\
\hline Algunas Veces & 3 & 2 & 0 & 1,7 & 28 & 26 & 26 & 22 & 24,7 & 26,5 \\
\hline Casi nunca & 0 & 0 & 0 & 0 & 0 & 39 & 42 & 41 & 40,7 & 43,7 \\
\hline Nunca & 0 & 0 & 0 & 0 & 0 & 19 & 18 & 19 & 18,7 & 20,1 \\
\hline TOTAL & 6 & 6 & 6 & 6 & 100 & 93 & 93 & 93 & 93 & 100 \\
\hline
\end{tabular}

Fuente: Elaboración propia

En la tabla 2, el 72\% de los directores aseguran que casi siempre colaboran con las actividades escolares, realizando tareas de forma colaborativa con los equipos de trabajo para unificar criterios y proponiendo estrategias que faciliten la colaboración entre todo el personal. Además, el $28 \%$ opina que algunas veces muestran estas competencias administrativas.

No obstante, el 43,7\% de los docentes considera que casi nunca los directores demuestran estas competencias en la institución, el 26,5\% opina que algunas veces lo hacen, pero el 20,1\% aseguró que nunca los directores gestionan como ellos lo plantean y, el 9,7\% dice que casi siempre lo hacen de ese modo.

Los datos anteriores indican igualmente que existen diferencias en la percepción de actitud entre directivos y docentes, por lo que se infiere que se presentan debilidades en las competencias administrativas, atendiendo a lo planteado por Aldape (2008), cuando explica que estas competencias constituyen los conocimientos y habilidades específicas que contribuyen a enlazar las actividades docentes con las demandas de la administración de la institución y del entorno de la misma. 
Tabla 3. Competencias Comunicativas

\begin{tabular}{|c|c|c|c|c|c|c|c|c|c|c|}
\hline \multirow{2}{*}{ Alternativas } & \multicolumn{5}{|c|}{ Directores } & \multicolumn{5}{|c|}{ Docentes } \\
\hline & $\begin{array}{l}\text { Item } \\
7\end{array}$ & $\begin{array}{l}\text { Item } \\
88 \\
\end{array}$ & $\begin{array}{l}\text { Item } \\
9\end{array}$ & $\mathbf{x}$ & $\%$ & Item 7 & $\begin{array}{l}\text { Item } \\
8\end{array}$ & $\begin{array}{l}\text { Item } \\
9\end{array}$ & $\mathbf{x}$ & $\%$ \\
\hline Siempre & 0 & 0 & 0 & 0 & 0 & 0 & 0 & 0 & 0,0 & 0,0 \\
\hline Casi siempre & 4 & 3 & 3 & 3,3 & 56 & 14 & 13 & 12 & 13,0 & 14,0 \\
\hline Algunas Veces & 2 & 3 & 3 & 2,7 & 44 & 26 & 19 & 21 & 22,0 & 23,7 \\
\hline Casi nunca & 0 & 0 & 0 & 0 & 0 & 27 & 39 & 34 & 33,3 & 35,8 \\
\hline Nunca & 0 & 0 & 0 & 0 & 0 & 26 & 22 & 26 & 24,7 & 26,5 \\
\hline TOTAL & 6 & 6 & 6 & 6 & 100 & 93 & 93 & 93 & 93 & 100 \\
\hline
\end{tabular}

Fuente: Elaboración propia

Muestra la tabla 3 que el $56 \%$ de los directores consideran que casi siempre expresan sus ideas con claridad para la toma de decisiones, exponen ideas innovadoras para la ejecución de proyectos institucionales y establecen diálogos abiertos para la planificación de objetivos. De la misma manera, el $44 \%$ opina que algunas veces actúa de esta manera, demostrando competencias comunicativas.

Por su parte, la mayoría de los docentes encuestados (35,8\% y $26,5 \%)$ indicaron que los directores casi nunca y nunca respectivamente muestran competencias comunicativas, mientras que el $23,7 \%$ considera que algunas veces los directores se comunican de forma efectiva, asimismo, el 14\% opina que casi siempre ocurre lo anterior.

Los datos arrojados por los docentes aquí analizados se corresponden con lo expuesto por Gorrochotegui (2010) quien refiere que los gerentes deben desarrollar mediante el uso de estrategias adecuadas, la competencia comunicativa, donde el lenguaje es el instrumento del intercambio de ideas, éstas, dan paso al conocimiento, si hay atención, dedicación, interés y comprensión de los mensajes, facilitando la 
Competencias del gerente educativo en instituciones educativas de Riohacha, Colombia.

interacción entre los sujetos a través de la comunicación eficaz en todos los departamentos de la organización educativa.

Tabla 4. Competencias Humano-Sociales

\begin{tabular}{|l|l|l|l|l|l|l|l|l|l|l|}
\hline \multirow{3}{*}{ Alternativas } & \multicolumn{4}{|c|}{ Directores } & \multicolumn{4}{c|}{ Docentes } \\
\cline { 2 - 13 } & $\begin{array}{l}\text { Item } \\
\mathbf{1 0}\end{array}$ & $\begin{array}{l}\text { Item } \\
\mathbf{1 1}\end{array}$ & $\begin{array}{l}\text { Item } \\
\mathbf{1 2}\end{array}$ & $\mathbf{X}$ & $\mathbf{\%}$ & $\begin{array}{l}\text { Item } \\
\mathbf{1 0}\end{array}$ & $\begin{array}{l}\text { Item } \\
\mathbf{1 1}\end{array}$ & $\begin{array}{l}\text { Item } \\
\mathbf{1 2}\end{array}$ & $\mathbf{X}$ & \multirow{2}{*}{} \\
\hline Siempre & 0 & 0 & 0 & 0 & 0 & 0 & 0 & 0 & 0,0 & 0,0 \\
\hline Casi siempre & 2 & 2 & 2 & 2 & 33 & 9 & 10 & 10 & 9,7 & 10,4 \\
\hline Algunas Veces & 4 & 4 & 4 & 4 & 67 & 25 & 27 & 27 & 26,3 & 28,3 \\
\hline Casi nunca & 0 & 0 & 0 & 0 & 0 & 34 & 35 & 31 & 33,3 & 35,8 \\
\hline Nunca & 0 & 0 & 0 & 0 & 0 & 25 & 21 & 25 & 23,7 & 25,4 \\
\hline TOTAL & 6 & 6 & 6 & 6 & 100 & 93 & 93 & 93 & 93 & 100 \\
\hline
\end{tabular}

Fuente: Elaboración propia

Se observa en la Tabla 4, referida a las competencias Humano-Sociales, que el 67\% de los directores asegura que algunas veces dialoga con sus colegas, ejecuta proyectos considerando las necesidades de la comunidad educativa y realiza sus labores con una visión optimista, también, el 33\% restante indicó hacerlo casi siempre. Sin embargo, el $35,8 \%$ de los docentes encuestados, junto a un $25,4 \%$ consideran que los directivos casi nunca y nunca demuestran competencias humano-sociales, mientras otro $28,3 \%$ señaló que algunas veces lo hacen y casi siempre según el 10,4\%.

Los resultados evidencian fallas, según la percepción del personal docente, ya que no se está promoviendo el trabajo armonioso con la comunidad educativa, tal y como lo sugieren Aldape (2008), para quien estas competencias humano-sociales constituyen los conocimientos y habilidades específicas relacionadas con la capacidad del docente para trabajar armoniosamente con su grupo de clase, sus compañeros, padres de familia y cualquier otra persona involucrada en el logro de su meta.

\section{Reflexiones Finales}

Una vez analizados y discutidos los resultados se puede afirmar que en cuanto al analizar las Competencias del Gerente Educativo en las instituciones de Riohacha, Colombia, se concluye que para los directores las competencias académicas, 


\section{Vianet Calle}

Telos Vol. 21, No. 3 (2019). 564-590

administrativas, comunicativas y humano-sociales, son puestas en práctica en las instituciones casi siempre, mientras que para los docentes estas competencias casi nunca son demostradas por el personal directivo, estas divergencias indican una sentida necesidad de mejoramiento de las competencias en el entorno escolar.

Todos estos aspectos han conllevado a replantearse una praxis pedagógica basada en competencias, para ello es necesario que todos los directivos y docentes tengan los conocimientos previos en cómo poder abordar todos los aspectos del currículo, la planeación de la información y cómo va a implementar los factores internos y externos influyentes en el ejercicio académico para lograr el éxito institucional y personal.

Por tal razón, en los escenarios actuales existe un reconocimiento del papel que la educación desempeña en los procesos de desarrollo, este se relaciona con la capacidad para hacer frente a los desafíos científicos-tecnológicos, para ponerse al día con la transformación productiva, esto implica, resolver problemas sociales y consolidar los procesos educacionales de manera competente.

Por consiguiente, cambiar la práctica gerencial implica perfilar un gerente diferente, con nuevos conocimientos, métodos y técnicas de abordaje y de solución de conflictos consonantes con el proceso de transformación e innovación característico del momento que por supuesto, deberá desprenderse de los esquemas tradicionales de supervisión gerencial para sumergirse en los roles actuales, con nuevos criterios, propuestas renovadas, desarrollándose un paradigma educativo innovador.

Se diría entonces, es urgente una transformación radical en el área educacional en el caso del Centro Educativo Enmanuel, Colegio Marcelo Graziosi y Gimnasio Campo David y que el gerente ocupe un lugar destacado, con participación activa y un compromiso ineludible, de superación y competencia profesional que aun cuando las poseen, no han sido incorporadas al ambiente del aprendizaje pedagógico de manera efectiva; puedan permitir a futuro, asumir un reto para impulsar una educación de 
Competencias del gerente educativo en instituciones educativas de Riohacha, Colombia.

calidad, requiriendo docentes competentes, con una necesidad de cambiar a través de un modelo pedagógico didáctico, construyendo nuevas estrategias gerenciales, integrando de forma reflexiva todas sus potencialidades y fortalezas.

\section{Referencias Bibliográficas}

Acevedo, Raziel. (2007). Factores que inciden en las competencias docentes universitaria un modelo jerárquico lineal. Tesis inédita de la Universidad Complutense de Madrid, Doctorado. Facultad de Educación, Departamento de Métodos de Investigación y Diagnóstico en Educación. Extraído de: https://eprints.ucm.es/7497/ Consulta: 14/10/2018

Acosta, Savier y Finol, Mineira. (2015). Competencias de los docentes de biología en las universidades públicas. Revista Telos, vol 17 (2), Venezuela. (Pp. 208224).

Aldape, Teresa. (2008). Desarrollo de las competencias del docente: Demanda De La Aldea Global Siglo XXI. Libros en red. España.

Alles, Martha (2015). Selección por Competencias. Ediciones Granica. Argentina. Arconada, Miguel (2006). Cómo trabajar con la publicidad en el aula. Competencia comunicativa y textos publicitarios. Grao. España.

Ávila, Raúl. (2013). La lengua y los hablantes. Trillas. México.

Belcher, Diane. \& Braine, George. (1995). Academic writing in a second language. Essays on Research and Pedagogy. Ablex Publishing Corporation. Estados Unidos.

Fajardo, Delia (2011). De la competencia comunicativa a la competencia intercultural: una propuesta teórica y didáctica para la educación intercultural en Latinoamérica. Revista ¿Educación intercultural. Escuela con calidad y equidad? Vol. 6 No 12. México. (Pp. 5-38)

García, Carlos Marcelo y Vaillant, Denise (2010). Desarrollo profesional docente: ¿Cómo se aprende a enseñar? Narcea. España. Extraído de: https://books.google.co.ve/books?isbn=8427716370 Consulta: 07/11/2018.

González, Margarita y Ramírez, Ignacio. (2011). La formación de competencias profesionales: un reto en los proyectos curriculares universitarios. Odiseo, revista electrónica de pedagogía, 8 (16). México. (Pp. 4-28)

Gorrochotegui, Alfredo. Un modelo para la enseñanza de las competencias de liderazgo. (2010) Revista Caminos Abiertos Educación y Educadores, [S.1.], v. 10, N $^{\circ}$ 2. México. (Pp. 87-102). 
Vianet Calle

Telos Vol. 21, No. 3 (2019). 564-590

Hellín, Pedro (2007). Publicidad y Valores Posmodernos. Editorial Siranda. España. Hurtado de Barrera, Jackeline (2008). Metodología y Técnica de Investigación aplicada a la comunicación. Panapo. Venezuela.

Martínez, Adrian (2005). Perfil de competencias del tutor de posgrado de la Universidad Nacional Autónoma de México. Primera edición. Editorial UNAM. México.

Miranda, Juan Leichtle, Patricia Montolio, Francisca y Cobin, John (2009). Análisis del nivel educacional de la población de la nueva región de los ríos, a través de una comparación intercensal (1992-2002): una mirada a las oportunidades laborales. Estud. Pedagógicos. Valdivia. Vol.35, no.2. Chile (Pp.105-122).

Moreno, Rosa; Menjívar, María; González, Paloma; Quan Vilma; Lainez, Ana; Monroy, Sonia; Guevara, Liliana; Tsung, Nien; Calderón, Rutilia; Córdova María; Córdova, Lorena; Jaramillo Vielka; Calvo, Oris; Caldera, Martha; Betango, Lieschen y de la Hoz, Gustavo (2011). Una mirada internacional a las competencias docentes universitarias. Investigación en primera persona: profesores y estudiantes. Primera edición. Ediciones Octaedro, S. L. España.

Moreno, Tiburcio. (2012). La evaluación de competencias en educación. Revista Sinéctica, (39), México. (Pp. 01-20).

Niño, Víctor. (2009). La Aventura de Escribir. Del pensamiento a la palabra. 1era edición. Editorial Ecoe ediciones. Colección Textos Universitarios. Colombia.

Palacino, Fredy (2007). Competencias comunicativas, aprendizaje y enseñanza de las ciencias naturales: un enfoque lúdico. Revista Electrónica de Enseñanza de las Ciencias. Vol. 6. No 2. España (Pp. 275-298).

Parlamento Europeo (2010). (Espacio Europeo Educación Superior). EEPS Extraído de: http://www.eees.es/ consulta: 14/07/2018.

Pozner, Pilar. (2000). Competencias para la Profesionalización de la Gestión

Educativa. IIPE - UNESCO Sede Regional. ANEP. Argentina.

Prego, Alberto (2007). La continua duda sobre qué y cómo enseñar: de la enseñanza reproductora a la educación social. Asociación Universitaria de Profesores de Didáctica de las Ciencias Sociales. Bilbao

Robbins, Stephen. (2009). Comportamiento Organizacional. Editorial Pearson Educación. México.

Semenov, Alexey (2005). Las Tecnologías de la Información y la Comunicación en la Enseñanza. Manual para docentes o Cómo crear nuevos entornos de aprendizaje 
Competencias del gerente educativo en instituciones educativas de Riohacha, Colombia.
abierto
por
medio
de las
TIC.
Extraído
de: https://unesdoc.unesco.org/ark:/48223/pf0000139028_spa.Consulta: $05 / 07 / 2018$.

Tobón, Sergio (2006). Aspectos básicos de la formación basada en competencias. Proyecto Mesesup. Extraído de: https://maristas.org.mx/gestion/web/doctos/aspectos_basicos_formacion_co mpetencias.pdf. Consulta: 10/07/2018

Tobón, Sergio (2011). Formación Basada en Competencia. Editorial ECOE. Colombia

Villarini, Angel. (2008). Desarrollo Humano basado en competencias. Una perspectiva crítica. De la pedagogía de la información para la adaptación a la antropogogía de la formación para la transformación. Extraído de: https://ofdpinternacional.com/wp-content/uploads/2018/10/Desarrollohumano-integral-basado.pdf. Consulta: 30/08/2018.

Zabalza Miguel. (2007). Competencias Docentes del Profesorado Universitario. Editorial Narcea. España. 